\title{
On boundary corrections of Lüscher-Weisz string
}

\author{
M. N. Khalil ${ }^{1,2,3,4, *}, A$. Bakry $^{5}, X . \mathrm{Chen}^{5}, M$. Deliyergiyev $^{6}, A . \mathrm{Galal}^{1}, A . \mathrm{Khalaf}^{1}$, and \\ P. M. Zhang ${ }^{7}$, \\ ${ }^{1}$ Department of Physics, Al Azhar University, Cairo 11651, Egypt \\ ${ }^{2}$ Department of Mathematics, Bergische Universität Wuppertal, Wuppertal 42097, Germany \\ ${ }^{3}$ Computation-based Science Research Center, Cyprus Institute, Nicosia 2121, Cyprus \\ ${ }^{4}$ Department of Physics, University of Ferrara, Ferrara 44121, Italy \\ ${ }^{5}$ Institute of Modern Physics, Chinese Academy of Sciences, Gansu 730000, China \\ ${ }^{6}$ Department of nuclear and particle physics, University of Geneva, CH-211, Switzerland \\ ${ }^{7}$ Department of physics, Sun Zhongshan University, Gaungdong 510275, China.
}

\begin{abstract}
The potential and the density profile of the QCD flux-tube are investigated within the framework of the Lüscher-Weisz (LW) string action with two boundary terms. The Numerical simulations involve 4D SU(3) Yang-Mills LGT at finite temperature. In general, we detect signatures of the two boundary terms considered in the LW string action. Near the end of QCD Plateau, the LW string is yielding a static potential which is in a good agreement with the lattice data for source separations $R \geq 0.3 \mathrm{fm}$. However, at $T / T_{c}=0.9$, the fit to the potential data improves with a good fit attained at $R \geq 0.7 \mathrm{fm}$. The mean-square width of the energy profile at $T / T_{c}=0.9$ matches well the width of the LW string over distance scales $R \geq 0.5 \mathrm{fm}$.
\end{abstract}

\section{Introduction}

The formation of a stringlike flux tube linking the color sources provides a prospective model for the confinement of quarks. In this picture, the nonperturbave properties of the QCD fluxtubes are expected to conform with that of an open effective bosonic strings in the long string limit. The model is expected to precisely predict a set of infrared (IR) observable which can be compared to the outcomes from the numerical lattice data [1].

Nevertheless, the analysis of string's fine structure in the lattice numerical data for the broadening profile revealed substantial mismatch [2] from the free-string Nambu-Goto (NG) model in the intermediate distance region at high temperatures. The excited spectrum [3] and string tension [4] obtained from the partition function of the leading-order Gaussian formulation of NG string action show a similar disagreement with the numerical data $[2,4,5]$ for source separation $R$ less than $1.0 \mathrm{fm}$.

The precise study of the strings effects suggests considering other features beyond the free NG action, in particular, possible stiffness properties [6] and boundary term corrections [7-9]. The Lorentz-invariant boundary corrections [9] to the static $Q \bar{Q}$ potential have been viable in modeling the data extracted either from the Wilson or the Polyakov-loop operators $[2,7,8]$.

The boundary corrections to the static quark potential may account for the deviations among predictions of the effective string and numerical outcomes [9]. Moreover, the contributions of the boundary action to the width profile have neither been theoretically calculated

\footnotetext{
*e-mail: m.nasr@stimulate-ejd.eu
} 
nor confronted with the numerical lattice data. These boundary effects are hoped to disclose the features of the profile of QCD flux-tube beyond the leading-order approximations which is the goal of this paper.

\section{Lüscher-Weisz string action}

The Lüscher and Weisz [1] action (physical gauge) which up to four-derivative term reads as

$$
\begin{aligned}
S^{\mathrm{LW}}[X] & =\sigma_{0} A+\frac{\sigma_{0}}{2} \int d^{2} \zeta\left[\left(\frac{\partial \boldsymbol{X}}{\partial \zeta_{\alpha}} \cdot \frac{\partial \boldsymbol{X}}{\partial \zeta_{\alpha}}\right)+\kappa_{2}\left(\frac{\partial \boldsymbol{X}}{\partial \zeta_{\alpha}} \cdot \frac{\partial \boldsymbol{X}}{\partial \zeta_{\alpha}}\right)^{2}+\kappa_{3}\left(\frac{\partial \boldsymbol{X}}{\partial \zeta_{\alpha}} \cdot \frac{\partial \boldsymbol{X}}{\partial \zeta_{\beta}}\right)^{2}\right]+\ldots \\
& +\gamma \int d^{2} \zeta \sqrt{g} \mathcal{R}+\alpha \int d^{2} \zeta \sqrt{g} \mathcal{K}^{2}+S^{b}
\end{aligned}
$$

the vector $X^{\mu}\left(\zeta_{0}, \zeta_{1}\right)$ maps the region $C \subset \mathbb{R}^{2}$ into $\mathbb{R}^{4}$. In the physical gauge $X^{1}=\zeta_{0}$ and $X^{4}=\zeta_{1}$ which restrict the string fluctuations to transverse directions of the world-sheet $C$. Invariance under party transform would keep only the terms of even number of derivatives. The map $g$ is the two-dimensional induced metric on the world sheet embedded in the background $\mathbb{R}^{4}$. The world-sheet area $A$ and the parameters $\sigma_{0}, \alpha$ and $\gamma$ are the string tension, the rigidity and the winding number, respectively. The couplings $\kappa_{2}, \kappa_{3}$ are effective low-energy parameters. The open-closed duality [3] imposes constraint on the kinematicallydependent couplings $\kappa_{2}, \kappa_{3}$ such that $\kappa_{2}+\kappa_{3}=\frac{-1}{8 \sigma_{0}}$. The above action Eq. (1) encompasses surface/boundary terms $S^{b}$ such that the symmetry breaking at the string's boundaries is built in. The boundary term $S^{b}$ is given by

$$
S^{b}=\int_{\partial \Sigma} d \zeta_{0}\left[b_{1} \frac{\partial \boldsymbol{X}}{\partial \zeta_{1}} \cdot \frac{\partial \boldsymbol{X}}{\partial \zeta_{1}}+b_{2} \frac{\partial \partial \boldsymbol{X}}{\partial \zeta_{1} \partial \zeta_{0}} \cdot \frac{\partial \partial \boldsymbol{X}}{\partial \zeta_{1} \zeta_{0}}+b_{3}\left(\frac{\partial \boldsymbol{X}}{\partial \zeta_{1}} \cdot \frac{\partial \boldsymbol{X}}{\partial \zeta_{1}}\right)^{2}+b_{4} \frac{\partial^{2} \partial \boldsymbol{X}}{\partial \zeta_{0}^{2} \partial \zeta_{1}} \cdot \frac{\partial^{2} \partial \boldsymbol{X}}{\partial \zeta_{0}^{2} \partial \zeta_{1}}\right]
$$

where $b_{i}$ are the couplings [3] of the boundary terms. Consistency with the open-closed string duality [3] implies a vanishing value of the first boundary coupling $b_{1}=0$, the leadingorder correction due to second boundary terms with the coupling $b_{2}$ appears at higher order than the four derivative term in the bulk. The last two geometrical terms are proportional to the extrinsic and intrinsic curvatures, respectively. We have discussed the geometrical and topological implications of these terms elsewhere [10, 11].

The static potential [12] at leading order reads

$$
V_{\ell \mathrm{o}}^{\mathrm{NG}}\left(R, L_{T}\right)=\sigma_{0} R+(d-2) L_{T} \log \eta(\tau)+\mu,
$$

where $\mu$ is a UV-cutoff and $\eta$ is the Dedekind $\eta$ function defined on the real axis as

$$
\eta(\tau)=q^{\frac{1}{24}} \prod_{n=1}^{\infty}\left(1-q^{n}\right)
$$

The static potential of NG string at second loop order is given by [13]

$$
V_{\mathrm{n} \ell \mathrm{o}}^{\mathrm{NG}}\left(R, L_{T}\right)=\sigma_{0} R+(d-2) L_{T} \log \eta(\tau)-L_{T} \log \left(1-\frac{L_{T}}{R^{3}} \frac{(d-2) \pi^{2}}{1152 \sigma_{0}}\left[2 E_{4}(\tau)+(d-4) E_{2}^{2}(\tau)\right]\right)+\mu,
$$

with $E_{2}$ and $E_{4}$ are the second and forth-order Eisenstein series. 
The boundary term $S^{b}$ in Lüscher-Weisz action accounts for the symmetry breaking by the cylindrical boundary conditions owing to the Polyakov lines. The modification to the potential received when considering Dirichlet boundary condition are given by [9]

$$
\begin{aligned}
V^{B} & =V^{b_{2}}+V^{b_{4}}, \\
V^{b_{2}} & =b_{2}(d-2) \frac{\pi^{3} L_{T}}{60 R^{4}} E_{4}(q), \\
V^{b_{4}} & =\frac{-b_{4}(d-2) \pi^{5} L_{T}}{126 R^{6}} \mathrm{E}_{6}(\tau) .
\end{aligned}
$$

The expectation value $[14,15]$ of the mean-square width of the free bosonic string theory in two dimensions

$$
W_{\ell o}^{2}\left(\zeta_{1}, \tau\right)=\frac{d-2}{2 \pi \sigma_{0}} \log \left(\frac{R}{R_{0}\left(\zeta_{1}\right)}\right)+\frac{d-2}{2 \pi \sigma_{0}} \log \left|\frac{\vartheta_{2}\left(\pi \zeta_{1} / R ; \tau\right)}{\vartheta_{1}^{\prime}(0 ; \tau)}\right|,
$$

where $\theta$ are Jacobi elliptic functions and $\tau=L / T$ is the modular paramter of the cylinder. The width due to the self-interaction is modified with the term $[15,16]$

$$
\begin{aligned}
W_{n \ell o}^{2}= & \frac{(d-2) \pi}{12 \sigma^{2} R^{2}}\left\{\tau\left(q \frac{d}{d q}-\frac{d-2}{12} E_{2}(\tau)\right)\left[E_{2}(2 \tau)-E_{2}(\tau)\right]-\frac{d-2}{8 \pi} E_{2}(\tau)\right\} \\
& +\frac{\pi}{12 \sigma R^{2}}\left[E_{2}(\tau)-4 E_{2}(2 \tau)\right]\left(W_{l o}^{2}-\frac{d-2}{4 \pi \sigma}\right) .
\end{aligned}
$$

The boundary term $S^{b}$ in Lüscher-Weisz action corrects the mean-square width $W_{b_{2}}^{2}, W_{b_{4}}^{2}$ [17] acoording to

$$
W_{b_{2}}^{2}=\frac{-\pi b_{2}(d-2)}{4 R^{3} \sigma^{2}}\left(\frac{1}{8}-\frac{1}{24} E_{2}(2 \tau)\right)
$$

and

$$
W_{b_{4}}^{2}=\frac{-\pi^{3}(d-2) b_{4}}{32 R^{5} \sigma^{2}}\left(\mathrm{E}_{2}(\tau)-\frac{5}{4}\right)\left(\frac{11 \mathrm{E}_{2}(\tau / 2)}{36}-\frac{5 \mathrm{E}_{2}(\tau)}{9}-\frac{55}{12}\right),
$$

respectively.

\section{Effective string lattice gauge theories}

\subsection{Simulation setup}

We choose to perform our analysis with lattices of temporal extents $N_{t}=8$, and $N_{t}=10$ slices at a coupling value $\beta=6.00$. The spatial size is $3.6^{3} \mathrm{fm}^{3}$ with lattice spacing $a=0.1$ $\mathrm{fm}$. The two lattices correspond to temperatures $T / T_{c}=0.9$ just before the deconfinement point, and $T / T_{c}=0.8$ which is near the end of QCD plateau.

The gauge configurations were generated using the standard Wilson gauge-action employing a pseudo-heatbath (FHKP) [18] updating to the corresponding three SU(2) subgroup elements (Marinari [19]). Each update step/sweep consists of one heatbath and 5 microcanonical reflections. The gauge configurations are thermalized following 2000 sweeps. The measurements are taken on 500 bins. Each bin consists of 4 measurements separated by 70 sweeps of updates. 


\subsection{Static quark potential}

The Monte-Carlo evaluation of the $Q \bar{Q}$ potential at source separation $R$ and temperature $T=$ $\frac{1}{L_{T}}$ is calculated through the expectation value of the Polyakov loop correlator

$$
\begin{aligned}
\mathcal{P}_{2 \mathrm{Q}} & =\int d[U] P(0) P^{\dagger}(R) \exp \left(-S_{w}\right), \\
& =\exp (-V(R, T) / T) .
\end{aligned}
$$

The time links is integrated out as detailed in Ref [20]. Making use of the space-time symmetries of the torus, the above correlator is evaluated at each point of the lattice and then

\begin{tabular}{|c|c|c|c|c|c|c|}
\hline & Fit Interval & Fit Param & $T / T_{C}=0.8$ & & & \\
\hline & $R \in I$ & $\sigma_{0} a^{2}$ & $\mu(\mathrm{LU})$ & $b_{2}(\mathrm{LU})$ & $b_{4}(\mathrm{LU})$ & $\chi_{\mathrm{dof}}^{2}$ \\
\hline & & & & & & \\
\hline$\div 2$ & {$[2,11]$} & $0.0422(1)$ & $-0.4767(6)$ & $-0.148(1)$ & 0.0 & 197.566 \\
\hline & {$[3,11]$} & $0.0452(2)$ & $-0.489(1)$ & $-0.607(9)$ & 0.0 & 5.91231 \\
\hline & & & & & & \\
\hline & {$[2,11]$} & $0.0452(3)$ & $-0.496(2)$ & $-0.44(2)$ & $0.10(1)$ & 1.37428 \\
\hline & {$[3,11]$} & $0.0450(4)$ & $-0.494(5)$ & $-0.3(2)$ & $0.02(0.1)$ & 1.17367 \\
\hline
\end{tabular}
averaged. We define the following possibly interesting combinations of LO and NLO solu-

Table 1. The $\chi^{2}$ values and the fit parameters; string tension and UV cutoff $(\sigma, \mu)$ together with $\left(b_{2}, b_{4}\right)$ returned from fits of data to boundary corrected potential at the LO of NG string with $T / T_{c}=0.8$. The potential $V_{\ell o}^{b_{2}}$ and $V_{\ell o}^{b_{2}, b_{4}}$ are given by Eq. (12) and Eq. (13), respectively.

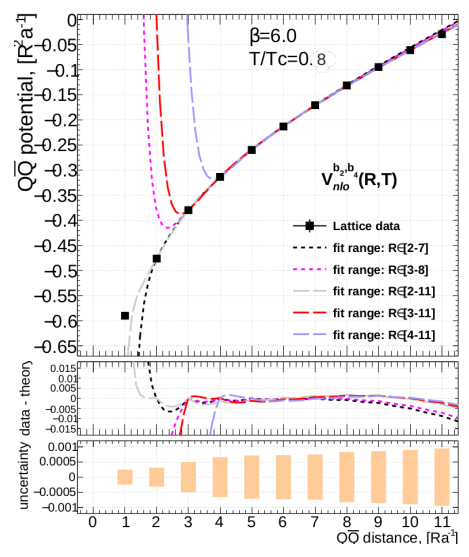

(a)

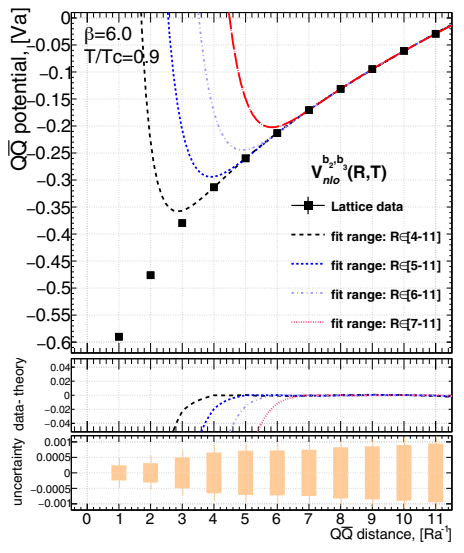

(b)

Figure 1. The $Q \bar{Q}$ potential at (a) $T / T_{c}=0.8$ and (b) $T / T_{c}=0.9$, the lines correspond to the NLO of NG string with two boundary terms $V_{n \ell o}^{b_{2}, b_{4}}$ Eqs. (12) and Eq. (13), respectively. 


\begin{tabular}{c|cccc} 
Fit Interval & \multicolumn{5}{|c}{ Fit Parameters, $T / T_{c}=0.9$} & & \\
$R \in I$ & $\sigma_{0} a^{2}$ & $b_{2}(\mathrm{LU})$ & $b_{4}(\mathrm{LU})$ & $\chi^{2}$ \\
\hline$\left[R_{m}, R_{M}\right]$ & & & & \\
{$[6,11]$} & $0.0401(3)$ & $11(4)$ & 0 & 363.1 \\
{$[7,11]$} & $0.03954(5)$ & $52(3)$ & 0 & 99.4 \\
{$[8,11]$} & $0.0389(2)$ & $205(50)$ & 0 & 9.4 \\
\hline$\left[R_{m}, R_{M}\right]$ & \multicolumn{5}{c}{} \\
{$[5,11]$} & $0.0400(3)$ & $1185.4(520.8)$ & $-948.9(417.7)$ & 336.6 \\
{$[6,11]$} & $0.0394(5)$ & $23246.0(14037.0)$ & $-18790.0(11352.0)$ & 89.2 \\
\hline
\end{tabular}

Table 2. The $\chi^{2}$ values and the corresponding fit parameters returned from fits to the NLO of NG string potential with boundary terms $V_{n \ell o}^{b_{2}}$ and $V_{n \ell o}^{b_{2}, b_{4}}$ given by Eq. (12) and Eq. (13) at $T / T_{c}=0.9$.

tions of Nambu-Goto string for the static potential with the boundary terms,

$$
\begin{aligned}
& V_{\ell o, n \ell o}^{b_{2}}=V_{\ell o, n \ell o}+V^{b_{2}}, \\
& V_{\ell o, n \ell o}^{b_{2}, b_{4}}=V_{\ell o, n \ell o}+V^{b_{2}}+V^{b_{4}},
\end{aligned}
$$

where subscripted $V_{\ell o, n \ell o}$ denotes either LO or NLO of the (NG) potential given according to Eq. (3) and Eq. (5). The $Q \bar{Q}$ potential data are fitted to either one of the boundary terms $V_{n \ell o}^{b_{2}}$ and $V_{n \ell o}^{b_{2}, b_{4}}$ given according to Eq. (12) and Eq. (13), respectively. The corresponding returned values of $\chi^{2}$ and fit parameters are enlisted in Table 1 at temperature $T / T_{c}=0.8$, and Table 2 at $T / T_{c}=0.9$, for the depicted fit intervals.

The analysis near the end of QCD plateau can disclose the substantial change in the properties of the confining force with the temperature. At the temperature $T / T_{c}=0.8$ where the thermal effects are significantly milder, the values of $\chi^{2}$ returned from the fits to $V_{\ell o}^{b_{2}}$ in Eq. (12) indicate minimal residuals of the fits over the interval $R \in[0.3,1.1] \mathrm{fm}$ with good $\chi^{2}=5.9$ taking into account the degree of freedom. Interestingly, the fits to the ansatz $V_{\ell o}^{b_{2}, b_{4}}$ with two boundary terms decrease the returned residuals significantly at a shorter interval of $R \in[0.2,1.1] \mathrm{fm}$

Figure 1(a) illustrates the fitted curves of the potential and the received small residuals over short distances as $R=0.3 \mathrm{fm}$ when the boundary term at NLO of NG string potentials. The plot reflects the small value of the fit residuals at all distances when considering $V_{n \ell o}^{b_{2}, b_{4}}$ which assumes close values to that enlisted in Table 1 . In the contrary to the fits at higher temperatures $T / T_{c}=0.9$ shown below the fit ansatz involving the string self-interaction Eq.(5) does not reduce the residuals over source separation $R<0.5 \mathrm{fm}$ [21]. Thus we may conclude that the boundary terms becomes the most relevant correction to the free string model with the decrease of the temperature.

The fit to the static potential $V_{n \ell o}^{b_{2}}$ at $T / T_{c}=0.9$ returns values for the parameter $b_{2}$ which appear to vary dramatically with the considered range. The values of $\chi^{2}$ are high when considering the entire intermediate fit-interval $R \in[0.5,1.1] \mathrm{fm}$. Nevertheless, the returned $\chi^{2}$ values in Table 2 appear to be much smaller than that obtained by considering merely the pure NG string potential Eqs. (5) (See Ref. [21]). Acceptable value of $\chi^{2}$ are returned over the fit interval $[0.7,1.2] \mathrm{fm}$ when considering the correction of both the two boundary terms $V_{\ell o}^{b_{2}, b_{4}}$. The fitted curves are plotted in Fig. 1(b) for the depicted ranges.

Inspection of Tables 1 and Table 2 indicates that fits to the NLO of the NG string with boundary term $V_{n \ell o}^{b_{2}}$ produce very close value of $\sigma_{0} a^{2}$ as that of the pure NG string $V_{n \ell o}$. The same observation holds for the fits considering two boundary terms $V_{n \ell o}^{b_{2}, b_{4}}$. Despite of 
the reduction in the minima of $\chi_{d o f}^{2}$, the same returned value [21] of the string tension fit parameter $\sigma_{o}$ is consistent with the modular transforms in Eq.(6), where the inverse of the cylinder's modular parameter does not produce terms linear in $R$ and do not contribute to the renormalization of the string tension.

The deviations of the string tension fit parameter from the values returned in Table 1 at $T / T_{c}=0.8$ is decreased considering other effects such as a possible rigidity property of the confing flux-tube. We reported in Ref [21] that the smoothed string fluctuations by virtue of the rigidity reproduce a close value of $\sigma_{0} a^{2} \sim 0.44$ returned at $T / T_{c}=0.9$.

\subsection{Energy-density profile}

The corrections provided by the boundary action to static $Q \bar{Q}$ potential seem to eliminate, to some extend, the deviations appearing when constructing the static mesonic states with Polyakov loop correlators. In the following we probe the confining force by profiling the action-density of the Yang-Mills vacuum in the presence of color charges. The action density is evaluated via a three-loop improved lattice field-strength tensor[2]. A scalar field characterization can be defined as

$$
C\left(\vec{\rho} ; \vec{r}_{1}, \vec{r}_{2}\right)=\frac{\left\langle\mathcal{P}_{2 Q}\left(\vec{r}_{1}, \vec{r}_{2}\right) S(\vec{\rho})\right\rangle}{\left\langle\mathcal{P}_{2 Q}\left(\vec{r}_{1}, \vec{r}_{2}\right)\right\rangle\langle S(\vec{\rho})\rangle}
$$

with the vector $\vec{\rho}$ referring to the spatial position of the energy probe with respect to some origin, and the bracket $\langle\ldots\rangle$ stands for averaging over gauge configurations and lattice symmetries.

We implement a number of $n_{s w}=20$ of improved cooling sweeps to eliminate statistical fluctuations which ought to keep the physical observable intact [2] over source separation upto $R \geq 0.5 \mathrm{fm}$. A double Gaussian fit function [2] is implemented to estimate the meansquare width. In analogy with the static $Q \bar{Q}$ potential, we examine same combinations of

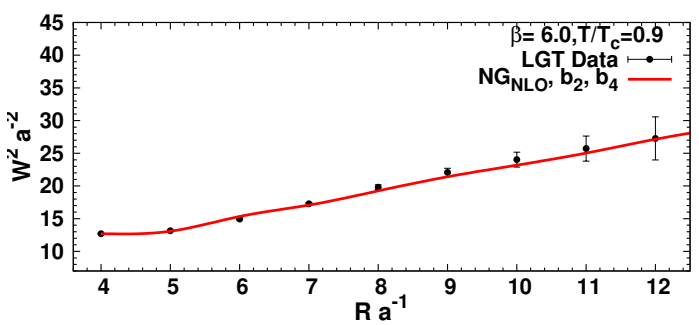

Figure 2. The mean-square width at the middle plane $R / 2$ and $T / T_{c}=0.9$. The solid line is the square-width of the effective NG string at NLO Eq. (7) and Eq. (8), with two-boundary terms $W_{b_{2}}^{2}+W_{b_{4}}^{2}$ Eq. (9) and Eq. (10) for fit range $R \in[0.4,1.2] \mathrm{fm}$.

boundary corrections (Eq. (9) and Eq. (10)) with both the leading and next-to-leading NambuGoto string's width defined by (Eq. (7) and Eq. (8)). The resultant $\chi^{2}$ and fit parameters are enlisted in the Tables. 3 and 4. The returned values in Table 3 indicates better match when including the first boundary correction $W_{b_{2}}^{2}$ Eq. (9) compared to the fits along the pure leading (NG) model [2]. The values of $\chi^{2}$ is further reduced upon switching on the string's self-interaction Eq. (8) together with the boundary corrections.

One can remark that the fits using merely the LO width from the NG string with $W_{b_{2}}^{2}$ boundary correction are providing smaller values of residual than that of the corresponding 


\begin{tabular}{|c|c|c|c|c|c|c|c|}
\hline \multirow{2}{*}{\multicolumn{2}{|c|}{ Plane, NG-order }} & \multicolumn{3}{|c|}{$R \in[0.5,1.2] \mathrm{fm}$} & \multicolumn{3}{|c|}{$R \in[0.6,1.2] \mathrm{fm}$} \\
\hline & & $\chi^{2}$ & $R_{0}$ & $b_{2}$ & $x^{2}$ & $R_{0}$ & $b_{2}$ \\
\hline \multirow{2}{*}{$z=1$} & $N G_{\ell o}$ & 52.6 & $13.5(3)$ & $12.8(6)$ & 14.98 & $16.1(5)$ & $22.2(1.6)$ \\
\hline & $N G_{n \ell o}$ & 5.7 & $13.9(3)$ & $-1.4(3)$ & 2.4 & $14.8(6)$ & $0.4(1.0)$ \\
\hline \multirow{2}{*}{$z=2$} & $N G_{\ell o}$ & 216.3 & 25.9 & $10.9(4)$ & 25.9 & $10.9(4)$ & $21.7(1.4)$ \\
\hline & $N G_{n \ell o}$ & 27.5 & 7.1(3) & $3.4(2)$ & 7.9 & $8.9(5)$ & 7.1(9) \\
\hline \multirow{2}{*}{$z=\frac{R}{2}$} & $N G_{\ell o}$ & 31.6 & $3.7(2)$ & $4.0(5)$ & 4.3 & $6.1(5)$ & $12.2(1.6)$ \\
\hline & $N G_{n \ell o}$ & 9.1 & $2.5(3)$ & $1.9(2)$ & 0.77 & $4.0(6)$ & $4.6(1.0)$ \\
\hline
\end{tabular}

Table 3. The returned $\chi^{2}$ and $b_{2}$ from the fits of string's width with boundary term $W_{b_{2}}^{2}$ Eq. (9).

\begin{tabular}{c|ccc|ccc}
\multicolumn{6}{c}{$R \in[0.5,1.2] \mathrm{fm}$} & \multicolumn{6}{c}{$R \in[0.6,12] \mathrm{fm}$} \\
Plane,NG-order & $\chi^{2}$ & $b_{2}$ & $b_{4}$ & $\chi^{2}$ & $b_{2}$ & $b_{4}$ \\
\hline$z=1, N G_{\ell o}$ & 52.2 & $33(31)$ & $-18(27.8)$ & 0.70 & $-121(38.0)$ & $131(34.8)$ \\
$N G_{n \ell o}$ & 5.66 & $-11(34.5)$ & $7(28.7)$ & 1.43 & $-36(36.7)$ & $31(30.8)$ \\
\hline$z=2, N G_{\ell o}$ & 92.5 & $62(27.2)$ & $-45(24.3)$ & 1.5 & $-153(35)$ & $161(32.5)$ \\
$N G_{n \ell o}$ & 27.5 & $4(30.8)$ & $-0.5(25.7)$ & 3.08 & $-67(34.1)$ & $63(28.7)$ \\
\hline$z=\frac{R}{2}, N G_{\ell o}$ & 24.20 & $137(29.9)$ & $-119(26.6)$ & 0.29 & $-59(49.9)$ & $67(46.3)$ \\
$N G_{n \ell o}$ & 6.96 & $108(35.7)$ & $-89(29.7)$ & 0.07 & $25(47.5)$ & $-17(40.3)$ \\
\hline
\end{tabular}

Table 4. Same as Table 3 for the boundary corrections $W_{b_{2}}^{2}+W_{b_{4}}^{2}$ given by Eq. (9) and Eq. (10).

pure NG string [2] at NLO. With the consideration of the two boundary corrections $W_{b_{2}}^{2}+$ $W_{b_{4}}^{2}$ a better match is retrieved over longer string length $R \geq 0.5 \mathrm{fm}$ (Table. 4). Figure 2 illustrates the fitted curves of the width growth at the middle plane resulting. The width of the string model employing the two boundary corrections $W_{b_{2}}^{2}+W_{b_{4}}^{2}$ provides good match with the LGT data even up to small source separation distances as $R=0.4 \mathrm{fm}$. Despite the relatively larger uncertainties in the density Eq. (14) compared to $Q \bar{Q}$ potential. However, the successful parameterization of the width profile is an indication of relevance to boundary action of confining flux tube at high energy.

\section{Conclusion}

In this work, the quark-antiquark potential and energy profile of a static meson is compared to the corresponding theoretical predictions laid down by the Lüscher-Weisz (LW) string with two boundary terms in the action. The static quark-antiquark $(Q \bar{Q})$ potential is calculated using link-integrated Polyakov loop correlators.

Near the end of QCD Plateau region, we detect signatures of the two boundary terms of the Lüscher-Weisz (LW) string in the Monte-Carlo lattice data. The boundary corrected string model is in good agreement with the static $Q \bar{Q}$ potential for color source separation as short as $R=0.3 \mathrm{fm}$. As we approach the deconfinement point $T / T_{c}=0.9$, the fits show reduction of the residuals by the inclusion of boundary terms of the Lüscher-Weisz action in the approximation scheme. However, deviations from the returned string tension $\sigma_{0} a^{2}$ persist.

For the energy width profile at $T / T_{c}=0.9$, the boundary corrected width with one boundary term for the (LW) action at two-loop order; or two boundary terms with (LW) action at 
one-loop order displays a good match with the LGT data for color sources separation $R \geq 0.5$ fm.

\section{References}

[1] M. Lüscher, P. Weisz, JHEP, 07, 049 (2002)

[2] A. S. Bakry, M. A. Deliyergiyev, A. A. Galal, A. M. Khalaf, M. N. Khalil, arXiv:heplat/2001.02392

[3] M. Lüscher P. Weisz, JHEP, 07, 014 (2004)

[4] O. Kaczmarek, F. Karsch, E. Laermann, M. Lutgemeier, Phys. Rev. D, 62, 3 (2000)

[5] KJ. Juge, J. Kuti, C. Morningstar, Phys. Rev. Lett., 90, 161601 (2003)

[6] H. Kleinert, Phys. Lett., B174, 335 (1986)

[7] M. Caselle, M. Panero, R. Pellegrini, D. Vadacchino, JHEP, 01, 105 (2015)

[8] BB. Brandt, JHEP, 02, 40 (2011)

[9] M. Billo, M. Caselle, F. Gliozzi, M. Meineri, R. Pellegrini, JHEP, 05, 130 (2012)

[10] A. S. Bakry, M. A. Deliyergiyev, A. Galal, M. N. Khalil, hep-th/1709.09446.

[11] A. S. Bakry, M. A. Deliyergiyev, A. Galal, M. N. Khalil, hep-lat/2005.04675.

[12] JF. Arvis, Phys. Lett., 127B, 106 (1983)

[13] K. Dietz and T. Filk Phys. Rev. D, 27, 2944 (1983)

[14] A. Allais, and M. Caselle, JHEP, 01, 073 (2009)

[15] F. Gliozzi, M. Pepe, UJ Wiese, Phys. Rev. Lett., 104, 232001 (2010)

[16] F. Gliozzi, M. Pepe, UJ. Wiese, JHEP, 11, 053 (2010)

[17] A. S. Bakry, M. A. Deliyergiyev, A. Galal, M. N. Khalil, hep-th/1912.13381

[18] K. Fabricius, O. Haan, Phys. Lett. B, 143, 459 (1984)

[19] N. Cabibbo and E. Marinari, Phys. Lett. B, 119, 387 (1982)

[20] P. deForcrand, C. Roiesnel, Phys. Lett., B151, 77 (1985)

[21] A. Bakry, M. Deliyergiyev, A. Galal, M. Khalil, hep-lat/2001.04203 